\title{
COLEOPTEROFAUNA VISITANTE DE Theobroma grandiflorum SCHUM. (STERCULIACEAE) DE UMA PLANTAÇÃO NOS ARREDORES DE MANAUS, AMAZONAS, BRASIL ${ }^{1}$
}

\author{
Maria das Graças Vale BARBOSA ${ }^{2}$; Claudio Ruy Vasconcelos da FONSECA
}

RESUMO - O cupuaçu (Theobroma grandiflorum Schumman), é uma planta frutífera nativa da Amazônia, com uma diversificação na entomofauna encontrada, registrando-se maior abundância na ordem Coleoptera. Em uma plantação nos arredores da cidade de Manaus usando-se métodos de coletas, manuais, com aplicação de inseticida (método de queda) e armadilhas de interceptação de vôo foram coletados durante 12 meses consecutivos, 1.212 exemplares de Coleoptera. Alguns aspectos em relação a fenologia e localização das plantas foram abordados. A maior abundância foi registrada em plantas localizadas em áreas de relevo plano expostas ao sol, com flores e sem frutos. Encontrou-se maior frequiência nos meses correspondentes ao período menos chuvoso (de junho a novembro), registrandose maior quantidade de exemplares no horário matutino. Foram identificadas 32 famílias e as mais abundantes foram Chrysomelidae $25,17 \%$, Curculionidae 18,08\%, (incluindo Scolytinae e Platypodinae), Staphylinidae $17,57 \%$ e Coccinellidae $12,46 \%$. Foram identificados indivíduos dos generos Palaminus (Staphylinidae), Phenrica, Asphaera, Colaspis, Homophoeta, Heilipus (Chrysomelidae), Desmobaris, Phitotribus, Comptocerus (Curculionidae) e das espécies Homophoeta aequinoctialis, Exora obsoleta, Spaethiella coccinea (Chrysomelidae), Colobothea hirtipes, Clorida curta. Compsibidion maronicum, Heterachthes pelonioides (Cerambycidae) e Marshallius multisignatus (Curculionidae). Os indivíduos de Spaethiella coccinea foram observados alimentando-se das folhas, Desmobaris e Heilipus foram encontrados alimentando-se dos brotos das folhas e das flores sendo considerados pragas para mudas das plantas. A relação dos indivíduos do gênero Phitotribus com a planta ainda não é conhecida.

Palavras-chave: Coleoptera, Theobroma grandiflorum, Método de Queda, Armadilhas de Interceptação de vôo, Amazônia.

Fauna of Coleoptera on Theobroma grandiflorum Schum. (Sterculiaceae) in Manaus, Amazonas State, Brazil

ABSTRACT - The cupuassu (Theobroma grandiflorum, Sterculiaceae) is a native Amazonian fruit tree that about its entomological informations had been demonstrated the highest insects diversity princi-pallyColeoptera. During in a period of 12 consecutive months, manual collections were realized using insecticide and flight interception traps in a private plantation of cupuassu in Manaus and 1.212 indi-viduals of Coleoptera visitors of Theobroma grandiflorum were collected. Some plants aspects phenologies were done. The most great abundance was registered in plants exposed to the sun, in plants with flowers and without fruits, and plants localized in level and steep areas. Seasonal differences were also considered, finding a greater frequency of samples in the months corresponding to the less rainy period (from June to Novem-ber). The quantity of samples collected (and the families of Coleoptera) was correlated with the period of collection, the morning hours presenting a greater sample quantity. From the Coleoptera found visit-ing the cupuassu plants, 32 families were collected and identified, the most abundant of which were Chrysomelidae $25.17 \%$, Curculionidae $18.08 \%$, Staphylinidae $17,57 \%$, Coccinellidae 12,46\%. The species Homophoeta aequinoctialis, Exora obsoleta, Spaethiella coccinea (Chrysomelidae), Colobothea hirtipes, Clorida curta,Compsibidion maronicum, Heterachthes pelonioides (Cereambycidae) and Marshallius multisignatus (Curculionidae), were identified. The samples of Desmobaris and Heilipus were found on leaves spronts and flowers of the plants being considered pest of young cupuassu plants, (Phytotribus), however their relation with the plant is not know yet. The Spaethiella coccinea (Chrysomelidae, Hispinae) was observed and collected feeding on leaves of the cupuassu plant.

Key words: Coleoptera, Theobroma grandiflorum, cupuassu, Amazonia

'Parte da dissertação de Mestrado apresentada ao programa de Pós-Graduação em Biologia

Tropical e Recursos Naturais do Convênio INPA/FUA

${ }^{2}$ Bolsista do INPA/CNPq e-mail:jgguerra@objetivonet.com.br

${ }^{3}$ CPEN/INPA. Caixa Postal 478, Manaus, AM. 69011-970 


\section{INTRODUÇAO}

O cupuaçu (Theobroma grandiflorum Schumman), é uma planta nativa da Amazônia, cujos frutos (os maiores do gênero) são considerados os melhores da flora regional (Agenda do CNPq, 1985), devido a sua rentabilidade como cultura perene, despertando interesse em seu cultivo para fins industriais, com demanda cada vez mais crescente. Durante os últimos anos, foram realizados alguns estudos sobre a fenologia, incluindo algumas informações sobre aspectos entomológicos principalmente envolvendo os insetos que causam danos a essa planta (Silva, 1976; Falcão \& Lleras, 1983; Trevisan \& Mendes, 1991; Barbosa, 1994; Venturieri, 1994; Barbosa, 1999). Da grande quantidade e variedade de insetos encontrados visitando essa planta, a ordem Coleoptera é a predominante. A despeito do grande número de insetos encontrados em cupuaçuzeiros e da abundância de Coleoptera visitando esta planta, os registros envolvendo esse tipo de fauna em particular, não são bem conhecidos. Por este motivo, realizou-se um levantamento dos coleópteros visitantes do cupuaçuzeiro, com o objetivo de contribuir com informações sobre as ações desses insetos nesta planta, abordando os vários aspectos como a composição de famílias e ou espécies, variação na abundância e as relações inseto - planta.

\section{MATERIAIS E MÉTODOS}

Este estudo foi realizado numa plantação de cupuaçú nos arredores de
Manaus numa área aproximada de $410.000 \mathrm{~m}^{2}$, localizada em floresta de terra firme, solo do tipo latossolo com relevos planos e inclinados, durante 12 meses consecutivos de fevereiro de 1992 a janeiro de 1993, desenvolvendo três métodos: coletas manuais, aplicação de inseticida e uso de armadilhas de interceptação de vôo.

Foram demarcadas ao acaso 36 plantas de porte médio, de um conjunto de cerca de 1000 pés de cupuaçu existentes na plantação, sendo, 12 para as coletas manuais e armadilhas de interceptação de vôo e 24 plantas para as aplicações de inseticida. Dessa forma, a cada mês durante os 12 meses de amostragem, em 3 plantas, foram realizadas observações e coletas de material, tomando os seguintes critérios: às plantas localizadas em áreas expostas ao sol, denominou-se plantas de área aberta, e plantas sombreadas, para as que ficavam cobertas por outras árvores ao seu redor; considerou-se ainda, plantas com e sem flores e frutos, de acordo com as observações tomadas durante as coletas.

As coletas manuais foram realizadas mensalmente durante 3 dias consecutivos, tomando-se 3 períodos divididos da seguinte forma: $1^{\circ}$. dia de 6:00 às $18: 00 \mathrm{~h}, 2^{\circ}$. dia de $06: 00$ às 12:00 h e $3^{\circ}$. dia de $12: 00$ às $18: 00 \mathrm{~h}$. Os coleópteros foram coletados em frascos de vidro, pequenos $(5,0 \mathrm{~cm} \times 1,0 \mathrm{~cm})$; rotulados de acordo com o horário e, posteriormente transferidos para pequenos frascos contendo alcool a $70 \%$.

Fez-se um censo geral mensal dos coleópteros adaptando-se o 
método do lençol de Bramante ("Knock down") (Abreu et al, 1989), substituindo-se o lençol por doze bandejas retangulares de PVC, com $0,63 \times 1,25 \mathrm{~cm}$ colocadas na base de cada árvore uma vez a cada mês (Barbosa, 1994). Usou-se um pulverizador manual para a aplicação do inseticida, piretróide de marca Cymperator a base de cipermetrina da ICI do Brasil. Esse tipo de inseticida, segundo o seu fabricante, oferece um amplo espectro de atividade, por ser biodegradável, não se acumula no meio ambiente, propiciando segurança aos seres humanos e aos animais. $\mathrm{O}$ inseticida foi aplicado uma vez pela manhã e outra pela tarde, em uma planta de área sombreada e outra de área exposta ao sol, aplicando-se no mesmo dia, ou em dias alternados em função das condições climáticas. $\mathrm{O}$ material coletado foi conservado em frascos com álcool a $70 \%$ etiquetados segundo o horário da aplicação.

Para as coletas noturnas utilizouse armadilhas de interceptação de vôo modificadas a partir das armadilhas de interceptação de vôo com cola (Chinag, 1973; Murphy, 1985). As armadilhas são constituídas de placas de acetato transparentes medindo 63 $\mathrm{cm} \times 125 \mathrm{~cm}$. As placas, foram fixadas com cordas de nylon ao redor da planta e classificadas em dois tipos: "armadilha adesiva" e "armadilha com frasco coletor". Na armadilha adesiva substituiu-se a cola por óleo 40 passado em toda extensão e nos dois lados das placas; sob as placas colocou-se canaletas de tubo PVC medindo $125 \mathrm{~cm}$ de comprimento e $4,5 \mathrm{~cm}$ de diâmetro, abertas e cortadas em toda a sua extensão, revestidas com uma tela de nylon servindo como peneira, para filtrar todo material entomológico que cai após bater na placa e escorrega devido ao óleo (Barbosa, 1994). O material foi recolhido com um pincel molhado em querosene (solvente), e conservado em um vidro com querosene sendo posteriormente transferido para um frasco com álcool a $70 \%$ adaptandose conforme os métodos de Murphy (1985). O segundo tipo de armadilha de interceptação de vôo com frasco coletor é formado por uma placa de acetato transparente, sem qualquer substância adesiva; sob a placa, fica um funil de plástico, com um frasco coletor contendo álcool a $70 \%$. Essas armadilhas foram colocadas ao redor da árvore uma vez por mês no período de 18:00 as 06:00 $\mathrm{h}$.

Todo o material coletado foi levado para o laboratório na Coordenação de Pesquisas em Entomologia (CPEN), onde os Coleoptera foram montados, etiquetados, identificados e depositados na coleção de invertebrados do INPA.

O material foi identificado conforme a classificação de Lawrence \& Newton (1995), no nível de família e subfamília usando-se chaves sistemáticas (Borror \& Delong, 1964; Costa Lima, 1952, 1953, 1955, 1956) e comparações com a coleção entomológica do INPA. As espécies da família Curculionidae foram identificadas pelo Dr. Sérgio A. Vanin da USP, as da família Cerambycidae pelo Dr. Ubirajara Martins do MZUSP e ainda, o gênero Palaminus da família Staphylinidae pelo Sr. Peter Hammond 
do Museu de História Natural de Londres.

Trabalhou-se com estatística não-paramétrica considerando-se $95 \%$ de intervalo de confiança; utilizou-se o teste de Mann-Whitney para medir variáveis independentes pareadas e não pareadas; "Spearman Rank Order Correlation" para correlações entre a quantidade de exemplares de coleópteros, e as presença ou ausência de flores e frutos, horário de coletas e fatores climáticos, conforme Zar (1984). Para normalização, os dados foram transformados para logaritmo na base dez $\left(\log _{10}\right)$.

\section{RESULTADOS}

Foram identificadas 32 famílias de coleópteros de um total de 1.212 indivíduos coletados. As cinco mais abundante foram, Chrysomelidae com 314 indivíduos representando 25,9\%, Curculionidae com 219 (18,1\%), Staphylinidae com $213(17,6 \%)$, Coccinellidae com $151(12,4 \%)$, Euglenidae com $71 \quad(5,8 \%)$, Nitidulidae com $45(3,7 \%)$ e as 26 famílias restantes com 199 exemplares equivalendo a $16,4 \%$, com uma densidade de 34 indivíduos de Coleoptera por planta (Tab. 1).

Em 4 das 32 famílias, foram identificados os gêneros e as espécies: Palaminus sp, na família Staphylinidae tendo sido o gênero mais abundante; Spaethiella coccinea, Exora obsoleta, Homophoeta aequinoctialis, Homophoeta sp, Phenrica sp, Colaspis sp, Asphaera sp, em Chrysomelidae; Colobothea hirtipes, Clorida curta e Compsibidion maronicum, Heterachthes pelonioides em Cerambycidae; Marshallius multisignatus, Heilipus sp, Desmobaris sp, Phitotribus sp e Comptocerus sp em Curculionidae (Tab. 2).

O maior número de indivíduos 581 , foi coletado com a aplicação de inseticida, representando $48,10 \%$, nas coletas manuais 386 , equivalendo a $31,68 \%$ e com armadilhas 245 $(20,21 \%)$, sendo $36(2,97 \%)$ nas armadilhas de interceptação de vôo com frasco coletor e $209(17,24 \%)$ nas armadilhas de interceptação de vôo com óleo adesivo (Fig. 1).

\section{Aspectos da fenologia e relação entre a abundância de Coleoptera visitante de cupuaçu e os critérios observados}

Observou-se a presença de flores durante o período de junho a dezembro registrando-se uma correlação negativa entre o período da presença de flores nas plantas e a precipitação $(\mathrm{r}=-0,54, \mathrm{P}=0,06)$, significando que as plantas florescem durante o período mais seco (Fig. 2). Encontrou-se frutos nos períodos de fevereiro a maio e de outubro a janeiro, apresentando uma correlação positiva entre o período da presença de fruto e a precipitação ( $\mathrm{r}=0,53, \mathrm{P}=0,06$ ) (Fig. 2). Registrou-se uma correlação positiva da abundância de Coleoptera coletados e o período de floração das plantas ( $\mathrm{r}=0,43, \mathrm{P}=0,009)$, (Fig. 3).

Coletou-se maior número de indivíduos de Coleoptera nas 
Tabela 1. Lista da composição de Coleoptera visitante de Theobroma grandiflorum (cupuaçu, Sterculiaceae) nos diferentes períodos de coleta.

\begin{tabular}{|c|c|c|c|c|c|c|c|c|}
\hline & \multirow[t]{2}{*}{ Familia } & \multirow[t]{2}{*}{ Subfamilia } & \multicolumn{4}{|c|}{ Número de indivíduos } & \multirow[t]{2}{*}{$\%$} & \multirow{2}{*}{$\begin{array}{c}\text { Média } \\
\text { individuos / } \\
\text { planta }\end{array}$} \\
\hline & & & Manhã & Tarde & Noite & TOTAL & & \\
\hline 1 & Anobiidae & & 4 & 14 & 1 & 19 & 1.57 & 0.53 \\
\hline 2 & Anthicidae & & 18 & 8 & 3 & 29 & 2.39 & 0.81 \\
\hline 3 & Biphylidae & & 0 & 0 & 3 & 3 & 0.25 & 0.08 \\
\hline 4 & Bostrichidae & & 0 & 0 & 3 & 3 & 0.25 & 0.08 \\
\hline 5 & Buprestidae & & 4 & 2 & 0 & 6 & 0.5 & 0.17 \\
\hline 6 & Cantharidae & & 1 & 2 & 0 & 3 & 0.25 & 0.08 \\
\hline 7 & Carabidae & & 2 & 2 & 5 & 9 & 0.74 & 0.25 \\
\hline \multirow[t]{2}{*}{8} & Cerambycidae & Cerambycinae & 1 & 0 & 1 & 2 & 0.17 & 0.06 \\
\hline & Cerambycidae & Lamiinae & 0 & 4 & 0 & 4 & 0.33 & 0.11 \\
\hline \multirow[t]{6}{*}{9} & Chrysomelidae & Alticinae & 69 & 54 & 1 & 124 & 10.2 & 3.44 \\
\hline & Chrysomelidae & Hispinae & 4 & 1 & 0 & 5 & 0.41 & 0.14 \\
\hline & Chrysomelidae & Cryptocephalinae & 4 & 5 & 0 & 9 & 0.74 & 0.25 \\
\hline & Chrysomelidae & Eumolpinae & 84 & 71 & 2 & 157 & 13 & 4.36 \\
\hline & Chrysomelidae & Galerucinae & 3 & 6 & 1 & 10 & 0.83 & 0.28 \\
\hline & Chrysomelidae & Bruchinae & 5 & 3 & 1 & 9 & 0.74 & 0.25 \\
\hline 10 & Coccinellidae & & 75 & 73 & 3 & 151 & 12.5 & 4.19 \\
\hline 11 & Colydidae & & 1 & 2 & 1 & 4 & 0.33 & 0.11 \\
\hline 12 & Corylophidae & & 3 & 4 & 7 & 14 & 1.16 & 0.39 \\
\hline 13 & Cryptophagidae & & 16 & 5 & 8 & 29 & 2.39 & 0.81 \\
\hline 14 & Cucujidae & & 1 & 2 & 1 & 4 & 0.33 & 0.11 \\
\hline \multirow[t]{9}{*}{15} & Curculionidae & Apioninae & 5 & 5 & 0 & 10 & 0.83 & 0.28 \\
\hline & & Baridinae & 9 & 4 & 0 & 13 & 1.07 & 0.36 \\
\hline & & Cryptorhychinae & 3 & 1 & 2 & 6 & 0.5 & 0.17 \\
\hline & & Erirhininae & 10 & 0 & 2 & 12 & 0.99 & 0.33 \\
\hline & & Erodiscinae & 0 & 1 & 0 & 1 & 0.08 & 0.03 \\
\hline & & Hylobiinae & 0 & 2 & 0 & 2 & 0.17 & 0.06 \\
\hline & & Tychiinae & 1 & 0 & 0 & 1 & 0.08 & 0.03 \\
\hline & & Otiorhynchinae & 11 & 8 & 4 & 23 & 1.9 & 0.64 \\
\hline & & Zygopinae & 5 & 6 & 0 & 11 & 0.91 & 0.31 \\
\hline
\end{tabular}


Tabela 1 - Continuação

\begin{tabular}{|c|c|c|c|c|c|c|c|c|}
\hline & \multirow[t]{2}{*}{ Familía } & \multirow[t]{2}{*}{ Subfamilia } & \multicolumn{4}{|c|}{ Nủmero de indivíduos } & \multirow[t]{2}{*}{$\%$} & \multirow{2}{*}{$\begin{array}{c}\text { Média } \\
\text { individuos / } \\
\text { planta }\end{array}$} \\
\hline & & & Manhã & Tarde & Noite & TOTAL & & \\
\hline & & Scolytinae & 2 & 1 & 8 & 0.66 & 0.22 & 0.11 \\
\hline & & Ipinae & 9 & 60 & 74 & 6.11 & 2.06 & 0.14 \\
\hline & & Platypodinae & 4 & 49 & 58 & 4.79 & 1.61 & 0.06 \\
\hline 16 & Euglenidae & & 45 & 22 & 4 & 71 & 5.86 & 1.97 \\
\hline 17 & Histeridae & & 2 & 1 & 0 & 3 & 0.25 & 0.08 \\
\hline 18 & Lampyridae & & 3 & 3 & 1 & 7 & 0.58 & 0.19 \\
\hline 19 & Lycidae & & 1 & 2 & 0 & 3 & 0.25 & 0.08 \\
\hline 20 & Lyctidae & & 0 & 2 & 1 & 3 & 0.25 & 0.08 \\
\hline 21 & Mordellidae & & 2 & 2 & 1 & 5 & 0.41 & 0.14 \\
\hline \multirow[t]{2}{*}{22} & Nitidulidae & Carpophilinae & 7 & 2 & 2 & 9 & 0.74 & 0.25 \\
\hline & & Nitidulinae & 6 & 4 & 26 & 36 & 2.97 & 1 \\
\hline 23 & Phalacridae & & 0 & 1 & 5 & 6 & 0.5 & 0.17 \\
\hline 24 & Ptilodactylidae & & 0 & 1 & 0 & 1 & 0.08 & 0.03 \\
\hline 25 & Ryzophagidae & & 0 & 2 & 7 & 9 & 0.74 & 0.25 \\
\hline \multirow[t]{3}{*}{26} & Scarabaeidae & Acanthocerinae & 0 & 0 & 1 & 1 & 0.08 & 0.03 \\
\hline & Scarabaeidae & Aphodinae & 0 & 1 & 2 & 3 & 0.25 & 0.08 \\
\hline & Scarabaeidae & Rutelinae & 0 & 1 & 0 & 1 & 0.08 & 0.03 \\
\hline 27 & Scydmaenidae & & 5 & 1 & 1 & 7 & 0.58 & 0.19 \\
\hline \multirow[t]{6}{*}{28} & Staphylinidae & Osoriinae & 0 & 1 & 0 & 1 & 0.08 & 0.03 \\
\hline & Staphylinidae & Steninae & 2 & 0 & 0 & 2 & 0.17 & 0.06 \\
\hline & Staphylinidae & Paederinae & 120 & 28 & 22 & 170 & 14 & 4.72 \\
\hline & Staphylinidae & Outros & 19 & 0 & 9 & 28 & 2.31 & 0.78 \\
\hline & Staphylinidae & Tachyporinae & 0 & 1 & 2 & 3 & 0.25 & 0.08 \\
\hline & Staphylinidae & Pselaphinae & 5 & 3 & 1 & 9 & 0.74 & 0.25 \\
\hline 29 & Tenebrionidae & Alleculinae & 3 & 5 & 0 & 8 & 0.66 & 0.22 \\
\hline Total & & & 581 & 386 & 245 & 1.212 & 100 & 33.7 \\
\hline
\end{tabular}


Tabela 2. Gêneros e espécies de Coleoptera encontrados em Theobroma grandiflorum (cupuaçu, Sterculiaceae)

\begin{tabular}{|c|c|c|c|}
\hline Familia & Subfamilia & Gênero/espécie & No. indivíduos \\
\hline \multirow[t]{7}{*}{ Chrysomelidae } & Alticinae & Phenrica sp & 1 \\
\hline & & Asphaera sp & 4 \\
\hline & & Homophoeta & 1 \\
\hline & & H. aequinoctialis & 1 \\
\hline & Galerucinae & Colaspis sp & 6 \\
\hline & Galerucinae & Exora obsoleta & 1 \\
\hline & Hispinae & Spaethiella coccinea & 5 \\
\hline Staphylinidae & Paederinae & Palaminus $s p$ & 165 \\
\hline \multirow[t]{4}{*}{ Curculionidae } & & Heilipus $\mathrm{sp}$ & 2 \\
\hline & Baridinae & Desmobaris $s p$ & 14 \\
\hline & Erirhininae & Phitotribus sp & 14 \\
\hline & & Marshallius multisignatus & 4 \\
\hline \multirow[t]{5}{*}{ Cerambycidae } & & Colobothea hirtipes & 2 \\
\hline & & Clorida curta & 2 \\
\hline & & Compsibidion maronicus & 4 \\
\hline & & Heterachthes pelonioides & 1 \\
\hline & & & 227 \\
\hline
\end{tabular}

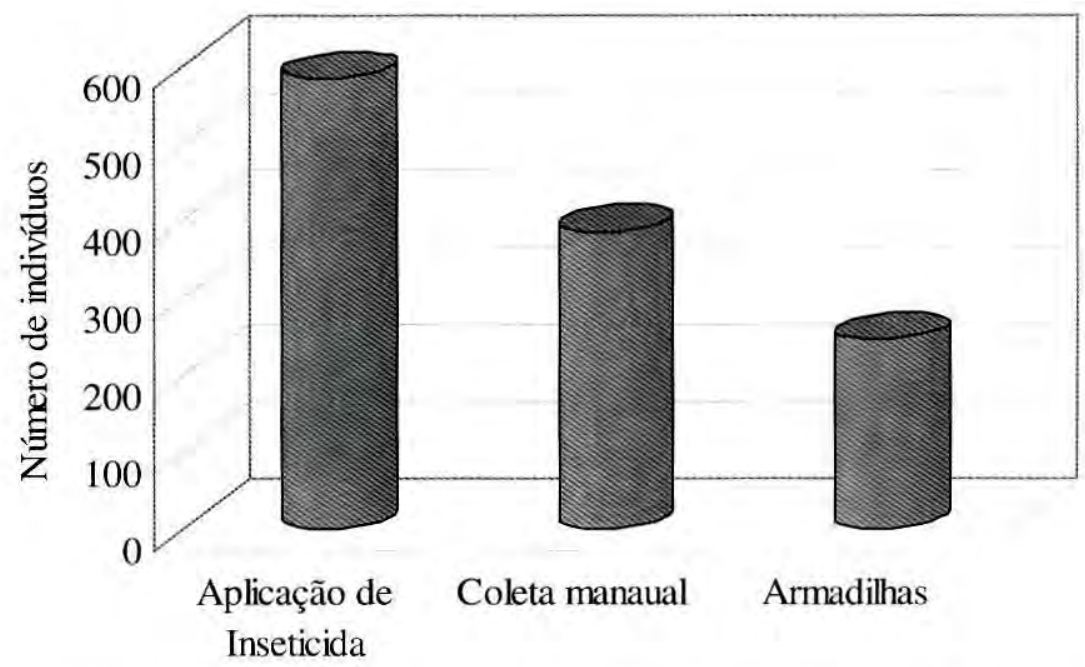

Figura 1. Número de indivíduos de Coleoptera coletados com diferentes métodos de amostragem, ( $\mathrm{CM}=$ Coleta manual, $\mathrm{AI}=$ Aplicação de inseticida, $\mathrm{AR}=$ armadilhas de interceptação de vôo), em Theobroma grandiflorum (Sterculiaceae). 
plantas sem frutos, com flores e localizadas em áreas abertas tanto de forma geral (Fig. 4), quanto em relação aos três métodos de amostragem registrando-se variações sem diferença significativa (Figs 5, 6 e 7).

\section{Aspectos da variação temporal da abundância de Coleoptera}

Coletou-se maior número de indivíduos durante o dia e a maior freqüência foi registrada pela manhã $48 \%$ (381) (Fig. 8). Durante o período diurno, as famílias de Coleoptera mais abundantes foram, Chrysomelidae, Staphylinidae e Curculionidae, (excluindo-se Scolytinae e Platipodinae) e no horário noturno Curculionidae (Scolytinae e Platypodinae) (Tab. 1).

O número total de indivíduos de Coleoptera ao longo dos doze meses amostrados apresentou uma distribuição multimodal (Fig. 9). Os meses de julho, agosto e setembro foram os mais representativo com 509 indivíduos representando $42 \%$ de todo o material de Coleoptera coletados e o mês de maio, o menos representativo com 31 indivíduos equivalendo a $2.55 \%$, observando-se o mesmo padrão de distribuição em relação aos métodos de coleta (Fig. 10). Registrou-se ainda, maior

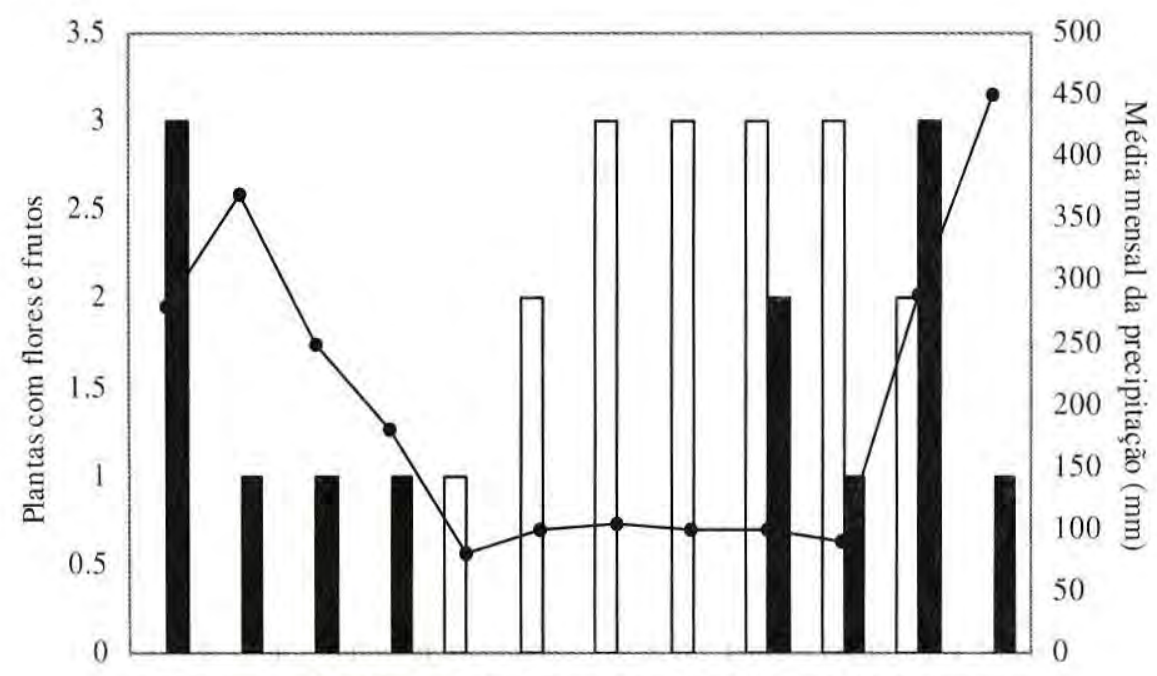

FEV MAR ABR MAI JUN JUL AGO SET OUT NOV DEZ JAN

$\square$ Flores $\square$ Frutos $\rightarrow$ Precipitação

Figura 2. Comparação entre o período de floração e frutificação registrado nas plantas de Theobroma grandiflorum (Sterculiaceae) observadas e a precipitação no período de fevereiro de 1992 a janeiro de 1993 em uma plantação nos arredores de Manaus, Amazonas, Brasil. 


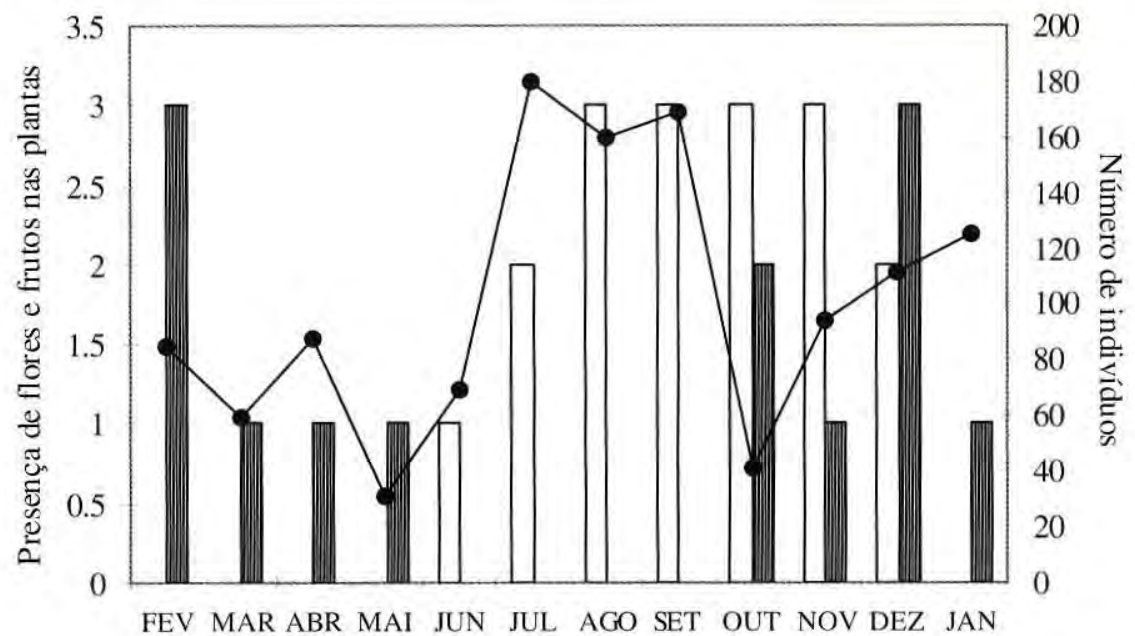

$\square$ Flores $\square$ Frutos $\longrightarrow$-Abundância

Figura 3. Comparação entre a abundância de Coleoptera e o período de floração e frutificação registrado nas plantas de Theobroma grandiflorum (Sterculiaceae) e a precipitação, no período de fevereiro de 1992 a janeiro de 1993 em uma plantação nos arredores de Manaus, Amazonas, Brasil.

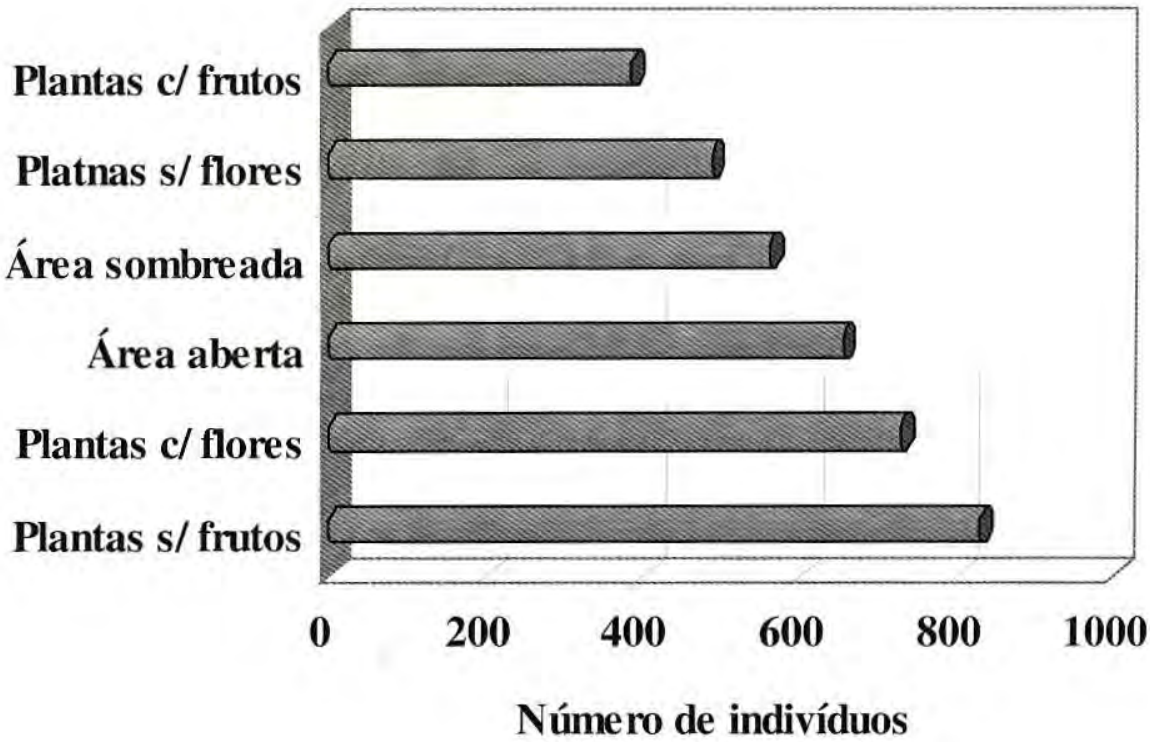

Figura 4. Número de indivíduos de Coleoptera coletados em plantas de Theobroma grandiflorum (Sterculiaceae) com e sem flores e frutos e localizadas em áreas expostas ao sol e sombreadas, durante o período de fevereiro de 1992 a janeiro de 1993 em uma plantação nos arredores de Manaus, Amazonas, Brasil.. 


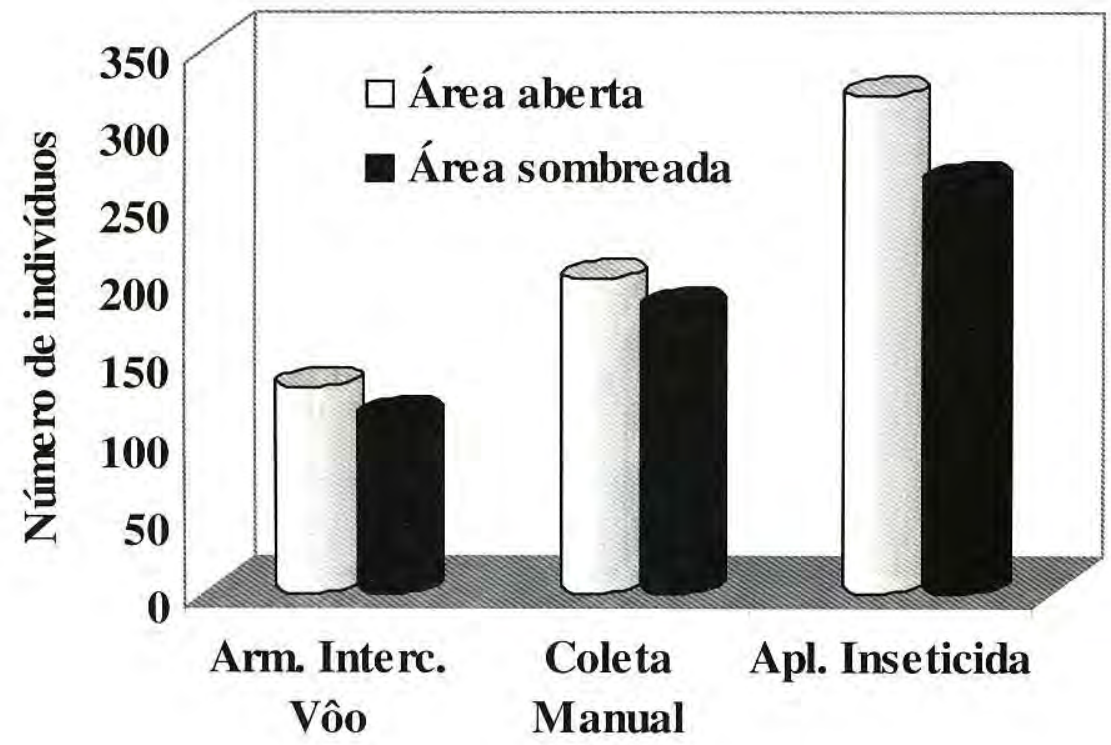

Figura 5. Número de indivíduos de Coleoptera coletados em plantas de Theobroma grandiflorum (Sterculiaceae) localizadas em áreas expostas ao sol e sombreadas, durante o período de fevereiro de 1992 a janeiro de 1993 em uma plantação nos arredores de Manaus, Amazonas, Brasil..

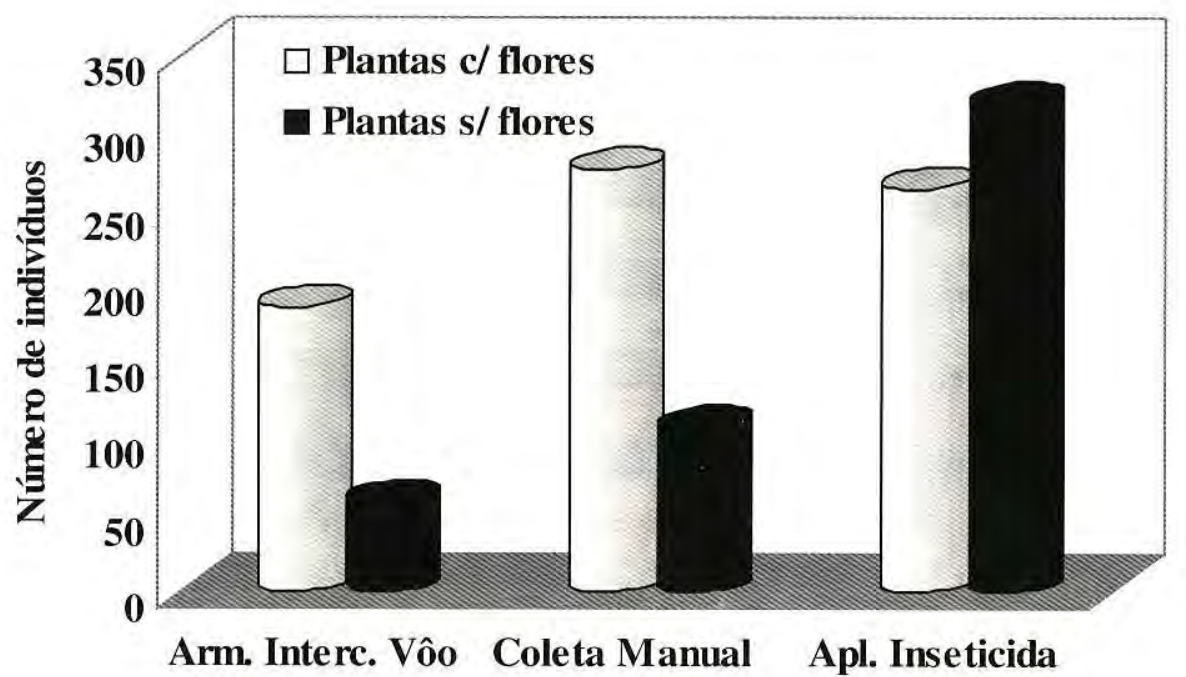

Figura 6. Número de indivíduos de Coleoptera coletados em plantas de Theobroma grandiflorum (Sterculiaceae) com presença e ausência de flores, com diferentes métodos de amostragens, durante o período de fevereiro de 1992 a janeiro de 1993 em uma plantação nos arredores de Manaus, Amazonas, Brasil.. 


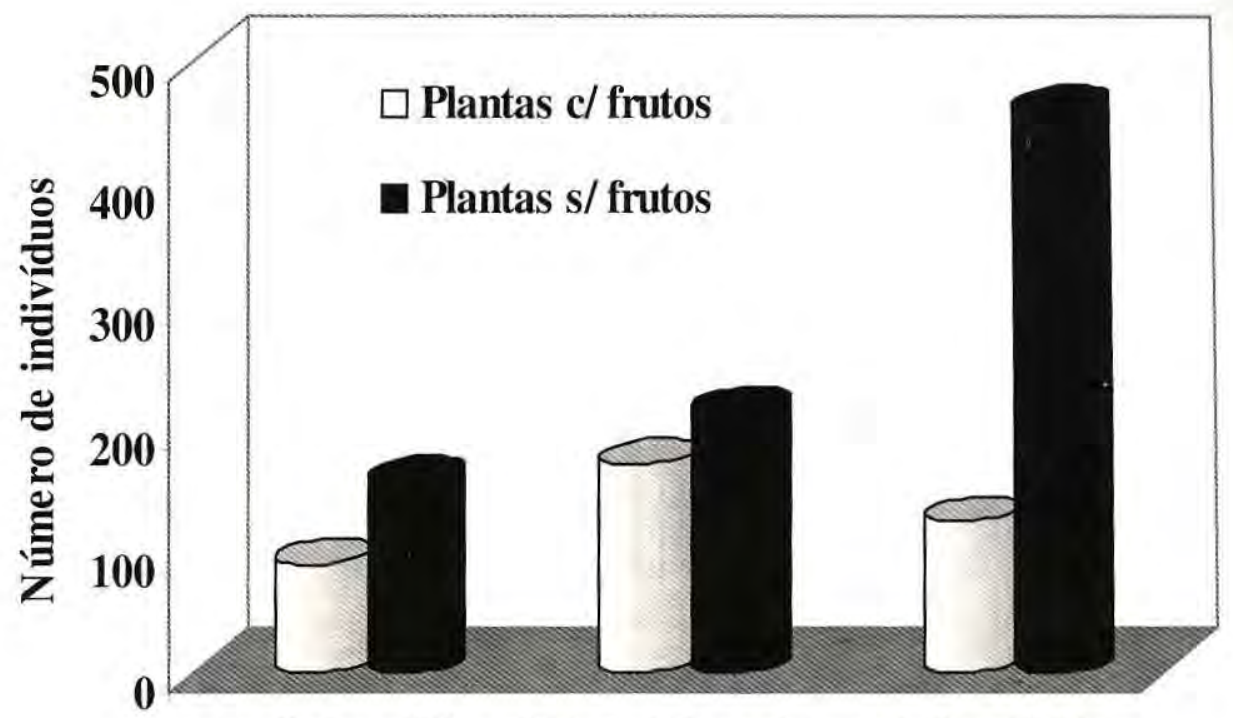

Arm. Interc. Vôo Coleta Manual Apl. Inseticida

Figura 7. Número de indivíduos de Coleoptera coletados em plantas de Theobroma grandiflorum (Sterculiaceae) com presença e ausência de frutos, com diferentes métodos de amostragens, durante o período de fevereiro de 1992 a janeiro de 1993 em uma plantação nos arredores de Manaus, Amazonas, Brasil..

número de indivíduos tanto no total quanto em relação aos diferentes métodos de coleta, durante os meses de junho a novembro, período considerado seco (Fig. 11).

\section{DISCUSSÃO E CONCLUSÃO}

A composição da fauna de Coleoptera visitante de plantas de cupuaçu apresentou maior abundância nas famílias Chrysomelidae e Curculionidae, grupos que incluem muitas espécies causadoras de danos a plantas (Bondar, 1939; Abreu, 1972, Garcia et al 1996; Mendes \& Garcia, 1982a), considerados os principais responsáveis por estragos no cacaueiro, pois destroem o limbo fo- liar, roem a casca de bilros e frutos, perfuram as partes ainda tenras dos caules, e quando atacam plantações novas, podem provocar retardamento no crescimento da planta comprometendo a produção. Espécies do gênero Colaspis e Spaethiella, alimentam-se de folhas (Abreu, 1972, Garcia et al 1996; Barbosa, 1999) e neste trababalho observamos que os indivíduos de espécies como Colaspis sp, Spaethiella coccinea, Desmobaris $\mathrm{sp}$, Heilipus sp e Camptocerus sp foram encontrados alimentando-se das folhas, flores e dos brotos das plantas.

A predominância desses grupos deve-se principalmente ao fato de que são famílias consideradas predominantementes fitófagas, cujas 

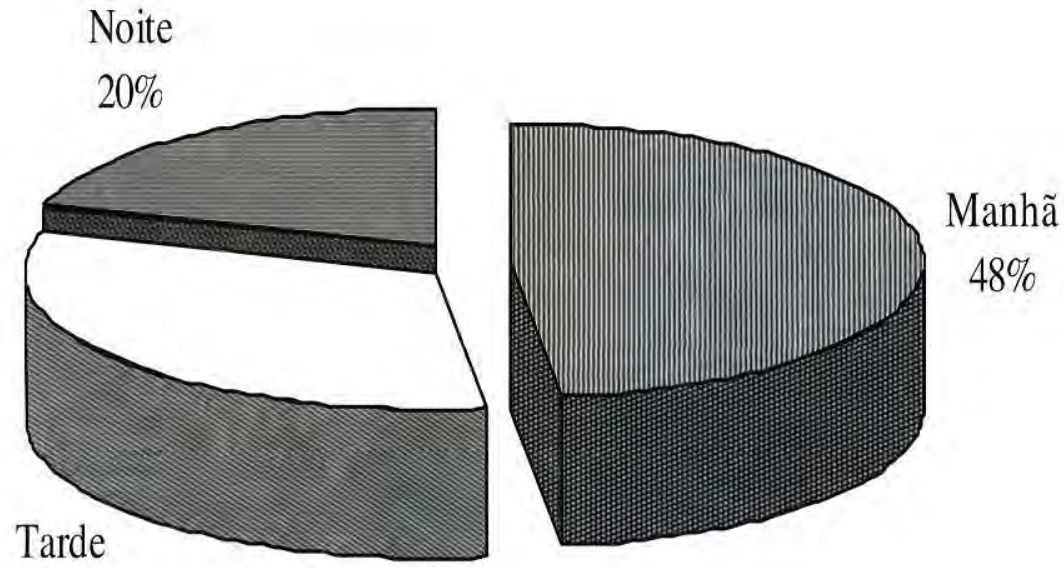

\section{$32 \%$}

Figura 8. Percentual do número de indivíduos de Coleoptera coletados em plantas de Theobroma grandiflorum (Sterculiaceae) nos diferentes horários de amostragem, durante o período de fevereiro de 1992 a janeiro de 1993 em uma plantação nos arredores de Manaus, Amazonas, Brasil..

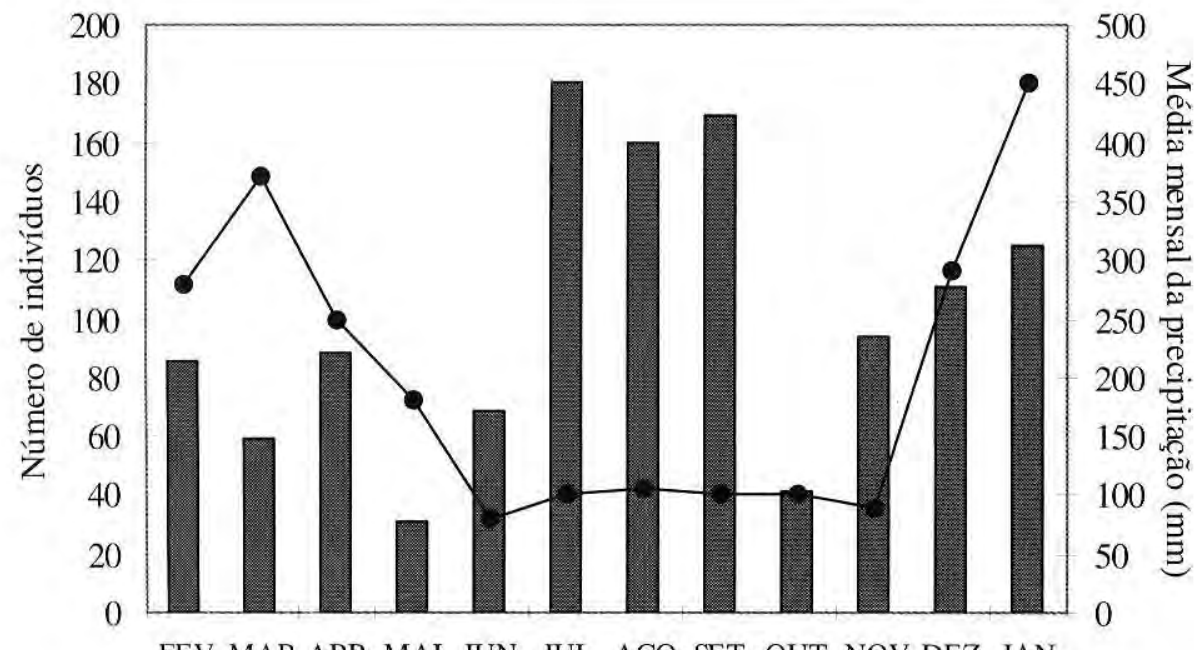

FEV MAR ABR MAI JUN JUL AGO SET OUT NOV DEZ JAN

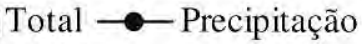

Figura 9. Distribuição do número de indivíduos de Coleoptera coletados em plantas de Theobroma grandiflorum (Sterculiaceae) durante o período de fevereiro de 1992 a janeiro de 1993 em uma plantação nos arredores de Manaus, Amazonas, Brasil.. 


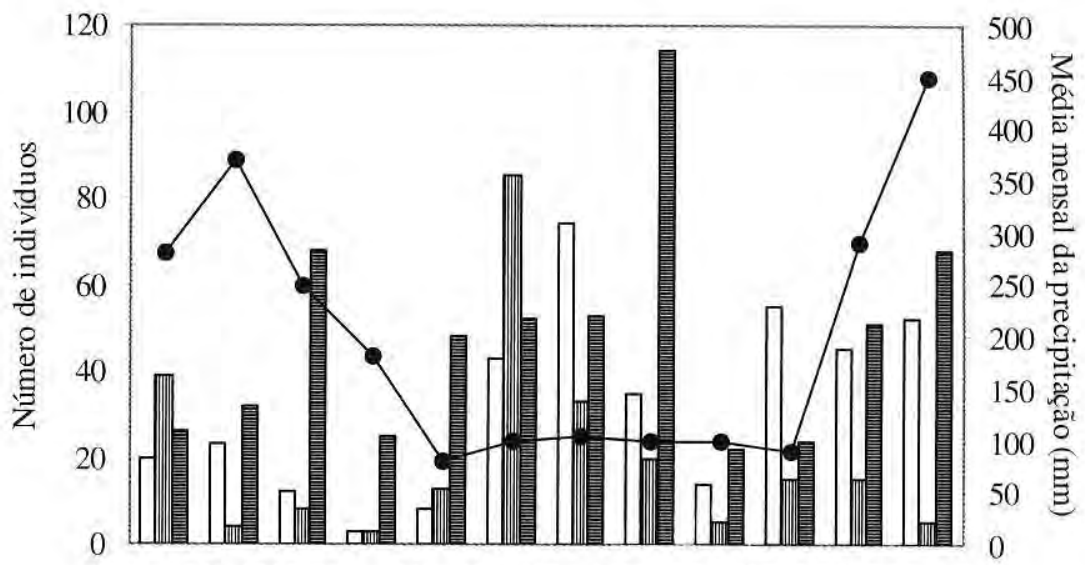

FEV MARABR MAI JUN JUL AGO SET OUT NOV DEZ JAN

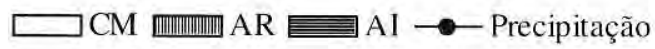

Figura 10. Correlação da abundância de Coleoptera coletados em plantas de Theobroma grandiflorum (Sterculiaceae), com diferentes métodos, durante o período de fevereiro de 1992 a janeiro de 1993 e a média mensal da precipitação, $(\mathrm{CM}=$ coleta manual, $\mathrm{AI}=$ aplicação de inseticida, $\mathrm{AR}=$ armadilhas de interceptação de vôo).

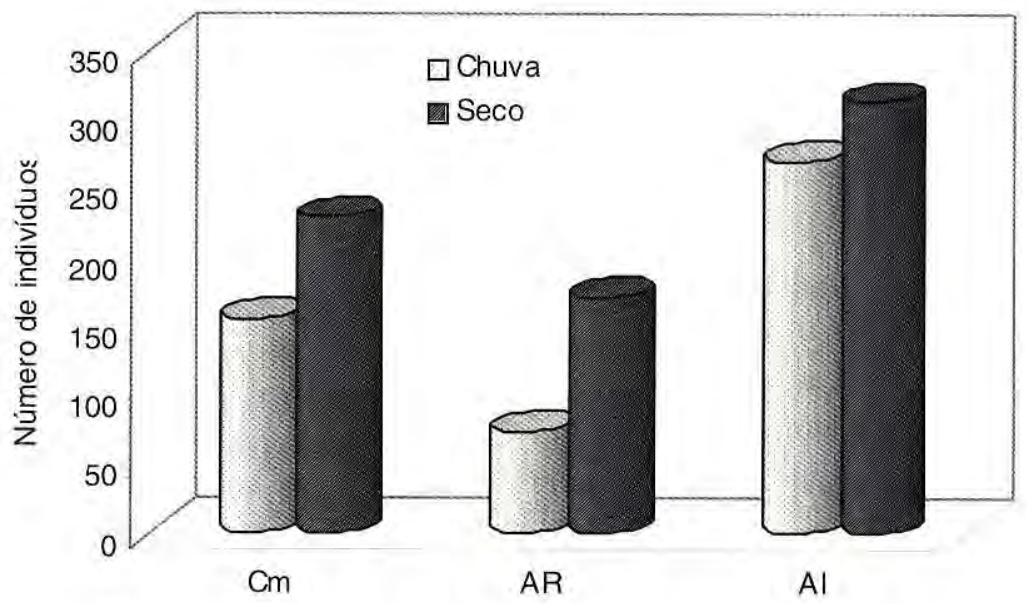

Figura 11. Número de indivíduos de Coleoptera coletados em plantas de Theobroma grandiflorum (Sterculiaceae) nos períodos de chuva e seca conforme os diferentes métodos de amostragens durante o período de fevereiro de 1992 a janeiro de 1993 e a média mensal da precipitação, ( $\mathrm{CM}=$ coleta manual, $\mathrm{AI}=$ aplicação de inseticida, $\mathrm{AR}=$ armadilhas de interceptação de vôo). 
espécies alimentam-se de partes diversas das plantas desde raiz, caule, folhas, brotos, flores e frutos (Bondar, 1939; Garcia \& Silveira Neto, 1980; Benton, 1984; Costa, Vanin \& CasariChen, 1988). Como são poucos os registros ou notificações de insetos visitantes ou pragas de $T$. grandiflorum (Silva, 1976; Trevisan \& Mendes, 1991; Venturieri, 1993, Barbosa, 1994; 1999), as informações sobre a entomofauna de cupuaçu obtidas durante este trabalho, mostraram que as famílias de coleópteros encontradas, são comuns a outras plantas, sejam fruteiras ou não, e dentre os insetos que se alimentam de plantas diversas como o cacaueiro, os coleópteros destacam-se como pragas importantes pela abundância e constância que ocorrem (Bondar, 1939; Abreu, 1972; Costa, 1977; Mendes \& Garcia, 1982a; Benton, 1984). Conforme resultado encontrado para Theobroma grandiflorum, pelo menos sete gêneros de Chrysomelidae e quatro gêneros de Curculionidae foram observados danificando partes essenciais da planta, tais como folhas, flores e brotos.

\section{Abundância em relação ao critérios observados}

\section{Plantas expostas ao sol, sombreadas, com e sem flores efrutos}

De acordo com Leite \& Muller (1983), plantas expostas ao sol emitem os maiores índices de floração e número de folhas. Registrou-se maior abundância de Coleoptera nas plantas localizadas em áreas expostas ao sol. As plantas de $T$. cacao lançam maior quantidade de folhas quando crescem sem sombra atraindo maior número de insetos fitófagos (Alvim, Machado \& Vello, 1972, apud Leite \& Muller, 1983). Segundo Alvim (1968), a emissão de folhas está diretamente relacionada com a produção da planta. Abreu (1972) estudando a flutuação de coleópteros nocivos ao cacaueiro concluiu que os lançamentos de folhas novas tiveram influência positiva altamente significativa com a flutuação da população de crisomelídeos e curculionídeos um e dois meses após sua ocorrência. Propôs ainda que os lançamentos foliares devem ser os indicadores para definir as épocas de combate às pragas. É provável que os insetos inclusive os coleópteros sejam atraídos até a planta pela quantidade de lançamentos foliares, caracterizando os insetos fitófagos, como destruidores do tecido das folhas (Mendes \& Garcia, 1982a). Neste estudo no entanto, os dados encontrados não corroboram a afirmação acima, tendo em vista que a maior abundância de Coleoptera foi registrado no período considerado seco, e em $T$. grandiflorum, segundo estudo feito por Falcão \& Lleras (1983), os lançamentos foliares ocorrem antes do início da floração, de janeiro à maio, ou durante a frutificação, período esse, com maior precipitação pluviométrica em que houve menor abundância. Curiosamente os dados mostram que maior número de exemplares de coleópteros foram coletados nas plantas com flores, portanto fora do período de lançamento foliar, conforme a citação acima. Segundo 
Calzavara (1987), o cupuaçuzeiro tem períodos de floração e frutificação simultâneos, de novembro à março com as flores aparecendo em junho e desaparecendo em março. De maneira geral a floração corresponde a época menos chuvosa, de junho a novembro (Falcão \& Lleras, 1983). Coleópteros como Chrysomelidae, Curculionidae, Anthicidae entre outros, desenvolvem hábitos fitófagos alimentando-se das estruturas florais (sépalas, pétalas, anteras) de várias plantas (Young, 1988). Outros insetos, são polinizadores, atraídos pelos odores emitidos durante o período de antese das flores, garantindo o sucesso na polinização, fator importante para a produção de frutos (Soria \& Wirth, 1974; 1981; Chenon, 1981; Genty, et al, 1986). Muitas famílias de hábitos florícolas e fungívoras foram encontradas neste trabalho tais como: Chrysomelidae, Mordellidae, Anthicidae, Euglenidae, Corylophidae, Cryptophagidae.

Durante o período de coleta foi observado que as plantas não apresentaram produção de flores de forma sincronizada, ou seja, grande parte das plantas marcadas para as coletas manuais e para a colocação das armadilhas de interceptação de vôo apresentaram flores durante o período de observação enquanto que a mais de $50 \%$ das plantas marcadas para a aplicação de inseticida não apresentaram flores. Dessa forma, é provável que, o registro do maior número de espécimes e de famílias de coleópteros encontrado nas coletas manuais e com armadilhas de interceptação de vôo se deva a presença de flores.

Outro fato observado foi que, mais da metade das plantas marcadas para as coletas, não apresentaram frutos durante o período assinalado na literatura como o da frutificação (Calzavara 1987). O baixo número de exemplares de Coleoptera coletados em plantas com fruto pode ser assim justificado pelo fato de que das plantas sorteadas, poucas apresentaram frutos durante o período de coletas.

\section{Aspectos da variação temporal da abundância de Coleoptera}

A maior frequência de indivíduos de Coleoptera foi registrada no período matutino e comparando-se o número de indivíduos coletados durante o período diurno e noturno, observou-se uma frequencia maior de indivíduos das subfamílias Scolytinae e Platypodinae durante o período noturno. Não podemos no entanto explicar com maiores detalhes o porque dessa preferência. Os levantamentos de entomofaunas visitantes de plantas como Theobroma cacao, Nerium oleander (Mendes \& Garcia, 1982b; Giannotti, Oliveira \& Machado, 1991) têm tido como objetivos principais, verificar a abundância dos insetos visitantes em diferentes horários, visando descobrir o comportamento destes insetos em relação a planta. No entanto, Giannotti, Oliveira \& Machado (1991), ao analisarem os dados obtidos sobre os insetos mais frequentes com os horários de visitas 
não encontraram significância, indicando que estas variáveis são independentes entre si.

Considerando-se que se registrou maior abundância de Coleoptera nos meses correspondentes ao período menos chuvoso (junho à novembro), acredita-se que essa diferença esteja ligada ao fato de que nesses meses ocorre a floração (Falcão \& Lleras, 1983), isso pode ser visto a partir da correlação negativa significativa do período de floração com a precipitação e a justificativa para que as flores sejam uma fonte de alimento para os indivíduos desse grupo, pode ser aceita tendo em vista maior número de indivíduos coletado em plantas com flores.

A despeito da menor quantidade de exemplares de coleópteros ter sido coletada nos meses correspondentes aos lançamentos foliares, coletou-se maior número de indivíduos em plantas localizadas em áreas expostas ao sol. Não ficou claro no entanto se o o aparecimento de folhas novas exercem ou não influência direta sobre a atração de coleópteros tendo em vista que não foi possível verificar esse fato no conjunto de plantas estudadas, o que indica necessidade de maiores observações em relação à fenologia desses vegetais.

\section{AGRADECIMENTOS}

Agradecemos ao Dr. José Albertino Rafael pela valiosa ajuda com o empréstimo da armadilha de interceptação de vôo e literatura, ao Sr. João Vidal (autor da adapatação da armadilha de interceptação de vôo), a Beatriz Ronchi-Telles pelo empréstimo do pulverizador de inseticida manual, a ICI do Brasil pela doação do inseticida e ao $\mathrm{Sr}$. Luís Aquino, pela ajuda nos trabalhos de campo.

\section{Bibliografia citada}

Abreu, J.M, 1972. Flutuações de populações de coelópteros nocivos ao cacaueiro no Espirito Santo. Revista Theobroma (Brasil) 2(1):45-55.

Abreu, J.M.; Nakayama, K.; Benton, F.P.; Cruz, P.F.N.; Ferraz, E.C.A.; Menezes, M. \& Smith, G.EF. 1989. Manejo de Pragas do Cacaueiro. CEPLAC/CEPEL. Ilheus, Bahia. 32p.

Agenda CNPq. 1985. Estrangeiros querem cupuaçu. 7(11):8.

Alvim, P.T. 1968. Fatôres responsáveis pela queda de produção de cacau na Bahia em 1968. Comunicação Técnica. 20.CEPLAC. Itabuna. Bahia.

Barbosa, M.G.V. 1994. Contribuição ao conhecimento da coleopterofauna visitante de cupuaçu (Theobroma grandiflorum [Willdenow ex Sprengel ]) Schumman em um bairro de Manaus Amazonas. Dissertação de Mestrado, Instituto Nacional de Pesquisas da Amazônia / Universidade do Amazonas. 143pp.

Barbosa, M.G.V. 1999. Occurrence of Spaethiella coccinea Boh. (Coleoptera :Chrysomelidae, Hispinae) on the Theobroma grandiflorum Schum. (Sterculiaceae) plants, in Manaus, Amazonas, Brazil. Acta Amazonica 29 (2): 313-317.1999

Benton, F.P. 1984 Abundância estacional dos coleópteros fitófagos do cacaueiro no sul da Bahia e no Espirito Santo. Revista Theobroma, 14(2) : 85-102.

Bondar, G. 1937. Curculionideos do gênero Conotrachelus nocivos ao cacaueiro. Rodriguesia, 2(2) : 41-43.

Bondar, G. 1939. Insetos nocivos ao cacaueiro. Instituto de cacau da Bahia. Boletim 
Técnico, no. 5, 112p.

Borror, D.J. ; Delong, D.M. 1964 Introdução ao Estudo dos Insetos. 1a. reimpressão, 1988. Editora Edgar Blucher Ltda. São Paulo. SP. Brasil. 653p.

Calzavara, B.B.G 1987 Cupuaçuzeiro. EMBRAPA/CPATU, Recomendações Básicas 1:5, 5p.

Chenon, R.D.1981 New data on the entomophil pollination of oil palm in West Africa. Advances in oil cultivation. Kuala Lumpur, Malasia, 47p.

Chiang, H. C. 1973. A simple trap for certain minute flying insects. Annals of the Entomological Society of America 66(3):704.

Costa Lima, A.M. 1952. Insetos do Brasil 7 (Coleópteros), Escola Nacional de Agronomia, Rio de Janeiro. 372p.

Costá Lima, A.M. 1953. Insetos do Brasil 8 (Coleópteros), Escola Nacional de Agronomia, Rio de Janeiro, 323p.

Costa Lima, A.M. 1955. Insetos do Brasil 9 (Coleópteros), Escola Nacional de Agronomia, Rio de Janeiro, 289p.

Costa Lima, A.M. 1956. Insetos do Brasil 10 (Coleópteros), Escola Nacional de Agronomia, Rio de Janeiro, 373p.

Costa, C.; Vanin, S.A.; Casari-Chen, S.A. 1988 Larvas de Coleóptera do Brasil. Museu de Zoologia, Ed. Universidade de São Paulo: FAPESP, 447p.

Falcão, M.A.; Lleras, E.1983. Aspectos fenológicos, ecológicos e de produtividade do cupuaçu (Theobroma grandiflorum Willd ex Spreng Schum.) Acta Amazonica, 13(5-6):725-735.

Garcia, J.J.S.; Silveira Neto, S. 1980. Estudos faunísticos de coleópteros e hemípteros associados ao cacaueiro no Estado do Pará. Revista Theobroma (Brasil) 10(1):15-23.

Garcia, M.V.B., Pamplona, A.M.S.R., Moraes, L.A.C.; Araujo, J.C.A.1996. Observaçôes sobre a biologia de Spaethiella tristis (Boh.) (Coleoptera: Chrysomelidae) e danos causados ao dendezeiro. An. Soc. Entomol. Brasil 25 (2):339-342.

Genty, P.; Garzon, A.; Lucchini, F. ; Delvare,
G. 1986. Polinizacion entomófila de la palma africana en America Tropical. Oleagineux, 41 (3) : 99-112.

Giannotti, E.; Oliveira, R.M.; Machado, V.L.L. 1991. Entomofauna visitante de Nerium oleander L. (Apocinaceae) durante o seu período de floração. BIOIKOS 5 (1) : 1-60

Lawrence, J.F.; Newton, Jr. A.F. 1995. Families and subfamilies of Coleoptera (With selected genera, notes, references and data on family - group numes. Pages 779-1006 in J. Pakaluk and S. A. Silpinski, editors. Biology, Phylogeny and classification of $\mathrm{Co}$ leoptera: papers celebrating the $80^{\text {th }}$ birthday of Roy A. Crowson. Museum i Instytut Zoologii PAN, Warszawa

Leite, R.M ; Muller, M.W. 1983. Influencia da remoção foliar sobre a fenologia de cacaueiros expostos ao sol e à sombra. Revista Theobroma, 13(3):203-210.

Mendes, A.C.B.; Garcia, J.J.S. 1982a. Flutuação populacional de crisomelídeos e curculionídios nocivos ao cacaueiro no polo de Rondônia, In Ilheus, BA, Brasil. CEPLAC/CEPEC. Informe Técnico.

Mendes, A.C.B. ; Garcia, J.J.S. 1982b. Ação de inseticida sobre insetos filofagos do cacaueiro e alguns predadores naturais na região de Ouro Preto (RO). In Ilheus, $\mathrm{Ba}$, Brasil CEPLAC/CEPEC. Informe Técnico. Ilheus. pp 295-296.

Mendes, A.C.B. ; Garcia, J.J.S. 1985. Ação de inseticidas sobre insetos nucivos e aranhas associados ao cacaueiro ná região de Ouro Preto dOeste, Rondonia, Brasil. Revista Theobroma 15(2) : 57-63.

Murphy, W.L. 1985. Procedure for the removal of insect specinens from sticky-trap inaterial. Annals of the Entomological Society of America 78(6): 881

Silva, M. F. 1976 Insetos que visitam o cupuaçu Theabrona grandiflorim (Willd ex Spreng) Schum (Sterculiaceae) e índice de ataque nas folhas. Arta Amazonica, 6(1):49-54

Soria, V.S.J. ; Wirth, W.W. 1974, Identidade e caracterizaçâo taxỗnica preliminar das mosquinhas Foripomya (Diptera, 
Ceratopogonidae) associadas com a polinização do cacaueiro na Bahia. Revista Theobroma, (Brasil) 4(1):3-12.

Soria, V.S.J.; Wirth, W.W. 1981. Ceratopogonid midges (Diptera; Nematocera) collected from cacao flowers in Caucagua, Miranda, Venezuela. Revista Theobroma, 11(4).

Trevisan, O. ; Mendes, A.C.B. 1991. Ocorrência de Conotrachelus humeropictus Fielder. 1940. (Coleoptera: Curculionidae), em frutos de cupuaçu, Theobroma grandiflorum (Willd. ex Spreng.) Schum. (Sterculiaceae). XIII Congresso Bras, de Entomol. Resumos, 1:137.

Venturieri, G.A. 1994. Floral Biology of cupuassu Theobroma grandiflorum (Willdenow ex Sprengel) Schumam. Ph.D. Thesis 211pp. University of Reading.

White, R.E. 1983. A field guide to the beetle of North America. The Peterson field guide series. 29.

Young, A.M. 1988. Notes on phenological patterns of flowering and flower feedeing beetles (Coleoptera: Chrysomelidae) in two clones of cacao (Sterculiaceae: Theobroma cacao L.) in Costa Rica. Turrialba 38(2) : 43-148.

Zar, R.J. 1984. Biostatistical Analysis. Prentice Hall. Second Edition. 717 pp. 